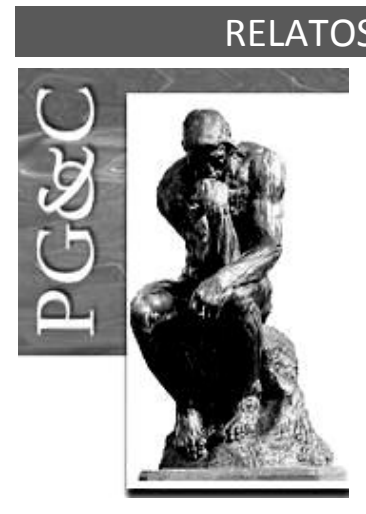

\title{
INTELIGÊNCIA COMPETITIVA EM CONTRATOS INTERNACIONAIS DE TECNOLOGIA: CONTRATAÇÕES DE UMA EMPRESA PETROLÍFERA
}

\author{
Fabíola de Moraes Spiandorello \\ Mestre em Engenharia de Materiais pela Universidade Federal de São \\ Carlos, Brasil. Doutoranda em Ciência, Tecnologia e Sociedade pela \\ Universidade Federal de São Carlos, Brasil. \\ E-mail: fabiolams@ufscar.br
}

Marcela Taiane Schiavi

Mestre em Ciência da Informação pela Universidade Federal de São Carlos, Brasil. Doutoranda em Ciência, Tecnologia e Sociedade pela Universidade

Federal de São Carlos, Brasil.

E-mail: tchela schiavi@yahoo.com.br

Wanda Aparecida Machado Hoffmann

Doutora em Ciência e Engenharia de Materiais pela Universidade Federal de São Carlos, Brasil. Professora da Universidade Federal de São Carlos, Brasil.

E-mail: wanda@ufscar.br

\begin{abstract}
Resumo
Os contratos internacionais de tecnologia que impliquem em remessa de valores ao exterior devem ser registrados junto ao Instituto Nacional da Propriedade Industrial (INPI). Essa exigência legal, aliada à necessidade de publicização de dados, originou uma base de dados pública que pode ser utilizada no contexto da Inteligência Competitiva (IC), posto que podem ser recobradas informações relevantes acerca das alianças estratégicas internacionais estabelecidas pelas empresas brasileiras visando à transferência de tecnologia. Sob essa ótica, as contratações internacionais de tecnologia realizadas pela empresa Petróleo Brasileiro S. A. (Petrobras) entre os anos de 2004 e 2018 foram monitoradas, sendo que mais de 840 extratos contratuais foram analisados. Tal análise permitiu a identificação dos fornecedores internacionais de tecnologia para a Petrobras, sua natureza e nacionalidade, valores investidos na aquisição da tecnologia, entre outras informações. Caracterizaram-se desta forma as alianças estratégicas estabelecidas tanto para a condução de atividades de transferência de tecnologia necessárias ao desenvolvimento da infraestrutura e operação de diversas plantas da companhia quanto para atividades de pesquisa, desenvolvimento e inovação (PD\&I). Aliado a isso, identificaram-se documentos patentários que suportaram alguns dos contratos de tecnologia analisados, aprofundandose a caracterização do modelo de contratação executado para aquisição de determinadas tecnologias. Com este estudo, demonstra-se que o uso dos registros contratuais disponibilizados em bases de dados públicas como fontes substanciais de informações relevantes podem auxiliar na ampliação da competitividade das empresas, já que tais informações podem ser obtidas e utilizadas para monitorar as atividades estratégicas de desenvolvimento tecnológico conduzidas por companhias que realizam inovação aberta.
\end{abstract}

Palavras-chave: Alianças Estratégicas. Inteligência Competitiva. Propriedade Intelectual. Transferência de Tecnologia. Contratos Internacionais.

Perspectivas em Gestão \& Conhecimento, João Pessoa, v. 8, número especial, p. 21-38, out. 2018. DOI: http://dx.doi.org/10.21714/2236-417X2018v8nep21

http://periodicos.ufpb.br/ojs2/index.php/pgc. ISSN: 2236-417X. Publicação sob Licença 


\title{
COMPETITIVE INTELLIGENCE IN INTERNATIONAL TECHNOLOGY CONTRACTS: CONTRACTING IN AN OIL COMPANY
}

\begin{abstract}
International technology contracts which give rise to remittance shall be registered by the Brazilian Patent Office (Instituto Nacional da Propriedade Industrial - INPI). This legal requirement, coupled with the legal statement for data publicity, gave rise to a public database which may be used as a source for Competitive Intelligence $(\mathrm{Cl})$, since important information may be retrieved concerning international strategic alliances established by Brazilian companies aiming technology transfer. In this context, international technology sourcing conducted by Petróleo Brasileiro S. A. (Petrobras) between 2004 and 2018 was monitored, with more than 840 contract extracts being analysed. This analysis allowed the identification of the international technology suppliers for Petrobras, nature and nationality of such organizations, amounts invested in the acquisition of such technologies and so on. This information allowed to understand the strategic alliances established both for conducting technology transfer activities regarding the development of infrastructure and operation of different industrial plants and for carrying on with research, development and innovation (RD\&l) activities. Besides that, one could identify patent documents which supported some of the technology contracts analysed, what allowed going deeper in the comprehension of the sourcing model carried out for the acquisition of certain technologies. This study demonstrates how contractual records made available in public databases may be used as important sources of relevant information which may help in increasing companies' competitiveness, since such information may be retrieved and used to monitor strategic activities regarding technological development conducted by companies which carry out open innovation.
\end{abstract}

Keywords: Strategic Alliances. Competitive Intelligence. Intellectual Property. Technology Transfer. International Contracts.

\section{INTRODUÇÃO}

A inovação tende a tornar-se um processo cujas etapas ocorrem não apenas em organizações distintas, mas particularmente em territórios diversos: países em desenvolvimento e desenvolvidos precisam se unir visando propor soluções para os desafios globais, unindo academia e indústria como direcionadores do crescimento econômico (WORLD..., 2015). De acordo com Criscuolo e Timmis (2017), as redes globais de produção do "mundo real" são redes complexas envolvendo fluxo de conhecimento, mercadorias e serviços, combinadas em vários estágios da produção. As empresas posicionadas no centro dessas complexas redes de produção têm acesso a uma maior variedade de insumos estrangeiros, o que afeta positivamente seu desempenho econômico. Segundo Hassani et al. (2017), tecnologia e inovação são necessárias na indústria de petróleo e petroquímicos para: i) produção e consumo sustentáveis do petróleo; ii) competitividade com outras indústrias; iii) automação de tarefas de elevado custo, perigosas e sujeitas a erros; iv) superação das questões relativas ao baixo preço do petróleo; v) garantir acesso a futuras fontes de óleo e gás.

Partindo-se dessa visão da necessidade contínua de inovação, integrada às Cadeias Globais de Valor (CGV), este estudo tomou por base a Petrobras por ser uma das empresas brasileiras que, além de desenvolver tecnologia proprietária, também se utiliza de tecnologia estrangeira em suas operações. Fundada em 1953, a Petrobras é uma empresa estatal brasileira de capital aberto, sendo classificada como uma NOC (National Oil Company). É a maior investidora em PD\&l entre as principais empresas petrolíferas globais e líder na exploração e produção em águas profundas e ultraprofundas (TORDO et al., 2011).

A Petrobras possui mais de 840 contratos de transferências de tecnologia, registrados junto ao INPI, dos quais faz uso sob a ótica da propriedade intelectual. Assim a contratação

Perspectivas em Gestão \& Conhecimento, João Pessoa, v. 8, número especial, p. 21-38, out. 2018. 
para transferência de tecnologia na Petrobras pode ocorrer de diversas maneiras: i) desenvolvimento do próprio recurso da empresa em uma determinada tecnologia, tornando-a pertencente apenas à Petrobras; ii) codesenvolvimento de uma tecnologia com recursos compartilhados entre a Petrobras e uma instituição ou organização; iii) aquisição de licença para que a Petrobras faça para uso de uma determinada tecnologia; iv) formação de joint ventures, nas quais a empresa se associa para fazer uso de uma determinada tecnologia em um período determinado; v) projeto multicliente, que envolve um projeto entre Petrobras e um grupo de pessoas que se reúnem para participar de pesquisas e desenvolver tecnologias relacionadas a elas; vi) aquisição de tecnologia, realizada com recursos próprios da Petrobras; e vii) open innovation, que nada mais é que o acesso a tecnologias disponíveis que não tenham restrições (DUARTE, 2013; PETROBRAS, 2012). Chesbrough (2006, p. 1) define open innovation como "o uso intencional de influxos e saídas de conhecimento para acelerar a inovação interna, e expandir os mercados para o uso externo da inovação, respectivamente". Tal paradigma "assume que a firma pode e deve utilizar tanto ideias externas quanto internas, bem como caminhos internos e externos para o mercado, conforme buscam avançar a tecnologia".

Calof (2018), ao identificar que a IC vem sendo cada vez mais formalizada junto às empresas europeias, afirmou que cada vez mais a inovação impulsiona as atividades de inteligência, particularmente em PD\&I e decisões relativas ao desenvolvimento de novos produtos. Por sua vez, Porter (1980) já defendia a necessidade de um processo estruturado de inteligência a qualquer tempo - e não apenas durante períodos de demanda intensa, como a identificação e estruturação para implementação de novos negócios - com o intuito de contínua e sistematicamente identificar as oportunidades e ameaças de negócios.

Assim, tendo por premissas a constante necessidade de inovação tecnológica para ampliação da competitividade, já em um contexto de inovação global - com participação centralizada na CGV de óleo e gás - realizou-se um estudo no qual, utilizando-se de informações públicas pertinentes à transferência internacional de tecnologia, foi possível a obtenção de uma visão geral das alianças estratégicas internacionais estabelecidas pela Petrobras, demonstrando a potencialidade do uso dessas informações como fonte de IC.

De posse dessas informações, torna-se possível elaborar um mapa da evolução relacional da companhia estudada, tendo por foco as parcerias internacionais formadas para PD\&I, podendo-se identificar os fornecedores de tecnologia, a quais classes as tecnologias que estão sendo desenvolvidas e transferidas pertencem, valores e prazos de absorção para a transferência dessas tecnologias etc. Em tempos de economia da inovação e do conhecimento, todas estas informações revestem-se de caráter extremamente estratégico para o monitoramento concorrencial e tomada de informações sobre mercados tecnológicos, entre outros aspectos, visando ao embasamento da tomada de decisões em empresas já que, conforme salientam Yusuf et al. (2014), a compreensão da dinâmica da competição na cadeia de fornecedores é mais importante para a empresa do que a análise das contendas entre empresas.

\section{REFERENCIAL TEÓRICO}

\subsection{Transferência de tecnologia}

De acordo com o Painel Intergovernamental em Mudança Climática (apud UNITED..., 2014 , p. 2) transferência de tecnologia é "um conjunto de processos que tratam da troca de conhecimento, dinheiro e mercadorias entre diferentes stakeholders o que leva à difusão de tecnologia para adaptação ou mitigação de mudanças climáticas". Se anteriormente a

Perspectivas em Gestão \& Conhecimento, João Pessoa, v. 8, número especial, p. 21-38, out. 2018. 
transferência de tecnologia era vista como uma forma de abrir mercados, atualmente ela é entendida como um processo que promove a difusão tecnológica, sendo conduzida entre organizações com o objetivo de aumentar sua competitividade por meio da inovação.

A transferência de conhecimento, ou de tecnologia, é formalizada por meio de tratados internacionais ou de acordos internacionais, sendo que as modalidades contratuais básicas são cessão (transferência de titularidade da propriedade industrial ou do know-how) ou licença de exploração (autorização de uso da propriedade industrial ou do know-how), podendo ou não ser combinados entre si (FLORES, 2008, p. 19).

O INPI (INSTITUTO..., 2017) reconhece as seguintes modalidades de contratos de transferência de tecnologia: i) licença de uso de marca (UM), sendo também possível a averbação de contratos de cessão de marcas; ii) licença de exploração de patentes (EP), sendo também possível a averbação de contratos de cessão de patentes; iii) licença de exploração de desenho industrial (EDI), sendo também possível a averbação de contratos de cessão de desenhos industriais; iv) fornecimento de tecnologia (FT), que objetivam a aquisição de conhecimentos ou técnicas que não são amparados por direitos de propriedade industrial (know-how); v) prestação de serviços de assistência técnica e científica (SAT), que estipulam as condições de obtenção de técnicas, métodos de planejamento etc. destinados à execução ou prestação de serviços especializados; e vi) franquia (FRA), que se destinam à concessão temporária de direitos que envolvam o uso de marcas, prestação de serviços de assistência técnica, combinadamente ou não, com qualquer outra modalidade de transferência de tecnologia necessária à consecução do objetivo.

\subsection{Alianças estratégicas}

Kanter (1994) originalmente definiu alianças estratégicas como relações entre empresas independentes para obter colaboração mútua e potencializar oportunidades. Li et al. (2013), ao estudarem alianças estratégicas internacionais, afirmaram que elas podem ser definidas como acordos voluntários entre empresas localizadas em países diversos, os quais envolvem a troca, o compartilhamento ou o codesenvolvimento de produtos, tecnologias ou serviços. Os benefícios alcançados com o estabelecimento de tais alianças incluem o compartilhamento de recursos, custos e riscos, barreiras de entrada a concorrentes mais fortes, colaboração para competição, aprendizado organizacional, ampliação da capacidade de inovação, legitimidade externa, entre outros. Estes benefícios vão ao encontro dos pilares estratégicos declarados pela Petrobras: compartilhamento de riscos, desoneração de investimentos, intercâmbio tecnológico, fortalecimento da governança, redução de custos operacionais, entre outros (PETROBRAS, 2018a).

Eiriz (2001), ao buscar desenvolver uma tipologia para alianças estratégicas, define que assistência técnica, subcontratação, acordos de pesquisa e desenvolvimento e de licenciamento de patentes podem ser classificados como alianças estratégicas do domínio técnico/produção.

Recentemente, Spiandorello e Hoffmann (2017) demonstraram a viabilidade da utilização dos contratos internacionais de transferência de tecnologia registrados junto ao INPI como fonte de identificação de alianças internacionais estratégicas entre empresas nacionais e universidades estrangeiras como esforço de PD\&I.

\subsection{Inteligência competitiva}

Os acordos internacionais de licenciamento de tecnologias são um dos mecanismos de transferência internacional de tecnologia plausível serem utilizados como elemento de

Perspectivas em Gestão \& Conhecimento, João Pessoa, v. 8, número especial, p. 21-38, out. 2018. 
monitoramento da informação (MI) e de Inteligência Competitiva (IC). Segundo Hoffmann (2011), MI é o método ou técnica de observação e acompanhamento sistemático e constante de dados, informação e conhecimento relevantes ao negócio da informação; o monitoramento dos concorrentes acompanha suas trajetórias, rotas estratégicas, desempenho e posicionamento competitivo; o monitoramento tecnológico permite o acompanhamento de fusões e aquisições visando à apropriação de tecnologia, bem como dos avanços técnicos, científicos e inovativos.

A IC pode ser definida como um processo de aprendizado, motivado pela competição, e baseada na informação que fortalece as estratégias da organização a curto, médio e longo prazo, a qual vem sendo incorporada nos processos de tomada de decisão de organizações públicas e privadas (HOFFMANN, 2011). E é uma atividade baseada na coleta de informações acerca de concorrentes através de meios legítimos, como dados públicos e entrevistas (CURTIS, 2001, p. 28).

\section{METODOLOGIA}

O estudo, de caráter quantitativo, apresenta principalmente a descrição dos resultados estatísticos obtidos a partir da análise dos dados coletados na Base de Dados do INPI. Visando à identificação de empresas estrangeiras que contratam com a Petrobras, realizou-se um levantamento de contratos internacionais de transferência de tecnologia junto à Base de Dados do INPI (INSTITUTO..., 2018a), na seção Contratos ("transferência de tecnologia"), derivada de dados publicados na Revista Eletrônica da Propriedade Industrial - RPI. Esta base de dados permite que as seguintes informações contratuais sejam recuperadas: partes contratantes; modalidade contratual; objeto do contrato; valor do contrato; moeda, valor e forma de pagamento; prazo de vigência do contrato; outras observações pertinentes (INSTITUTO..., 2017). As demais informações contratuais, cujo caráter é sigiloso, não se encontram disponibilizadas.

Foram utilizados os seguintes termos de busca, inseridos no campo "termos do certificado": petrobras (845); petróleo brasileiro (836); petrobras and PI (10). Os números entre parênteses indicam a quantidade de resultados retornados pela respectiva chave-debusca. Foram retidos 822 contratos de interesse; registros pertinentes a contratos nacionais ou nos quais a empresa em questão não configurava como cessionária (ou interveniente) foram expurgados.

A partir dos dados constantes dos contratos-tipo EP e/ou EP/FT identificados, buscaram-se as informações patentárias pertinentes aos documentos utilizados nesses instrumentos contratuais. Foram identificadas 21 patentes que foram licenciadas ou sublicenciadas para a Petrobras. Os dados pertinentes à titularidade, data de depósito, país da prioridade, família de patentes e classificação internacional foram obtidos consultando-se a Base de Dados do INPI (INSTITUTO..., 2018b), na seção Patentes, e a Base de Dados Espacenet, disponibilizada pelo Escritório Europeu de Patentes (EUROPEAN..., 2018). As informações contratuais foram cruzadas com as informações patentárias, de modo a obterem-se novas informações acerca de outros elementos integrantes das alianças estratégicas estabelecidas pela Petrobras.

O cerne dos procedimentos adotados para a recuperação tanto dos registros contratuais quanto dos patentários foi elaborado manualmente e organizado em planilhas eletrônicas. Tais procedimentos permitiram a extração de informações para este estudo, possibilitando a análise isolada de cada dado contratual e patentário relevante, bem como seu cruzamento, aprofundando-se a compreensão dos processos de transferência internacional de tecnologia conduzidos pela Petrobras.

Perspectivas em Gestão \& Conhecimento, João Pessoa, v. 8, número especial, p. 21-38, out. 2018. 


\section{RESULTADOS}

Os 822 registros contratuais retidos e analisados permitiram construir um perfil das contratações internacionais de transferência de tecnologia realizadas pela Petrobras entre os anos de 2004 (primeiro ano em que há dados disponibilizados da empresa) e junho de 2018, conforme se depreende do gráfico $a$ da Figura 1.

A variação no número de contratações internacionais coincide com o perfil de investimentos (totais e em PD\&l) realizados pela empresa no mesmo período, conforme se depreende do gráfico $b$ da mesma Figura 1. É válido também ressaltar que no ano de 2018 não houve a publicação pelo INPI de nenhum registro pertinente a contratações internacionais realizadas pela companhia.

O grau de endividamento da companhia foi significativamente ampliado a partir de 2008, sendo que em 2015 atingiu o ápice, quando a dívida líquida era mais de cinco vezes superior ao Lajida $^{1}$ (PETROBRAS, 2018b). Em 03 de Dezembro de 2012, foi aprovada pelo Conselho de Administração da companhia a criação de um programa de desinvestimentos (AYRES, 2012), visando à recuperação financeira da Petrobras, o qual começou a ser implementado como o Programa de Parcerias e Desinvestimentos a partir de 2015, sendo a principal ferramenta para diminuir a alavancagem da empresa. Os efeitos do Programa de Parcerias e Desinvestimentos, aliados à diminuição de investimentos e de custos operacionais, entre outros, auxiliam a explicar a queda no número de contratos internacionais de transferência de tecnologia firmados pela Petrobras a partir de 2012.

Figura 1 - a) Distribuição anual do número de protocolos de pedidos de averbação ou registro de contratos de transferência de tecnologia junto ao INPI. b) Distribuição anual do volume de investimentos, totais e em PD\&I, realizados pela Petrobras

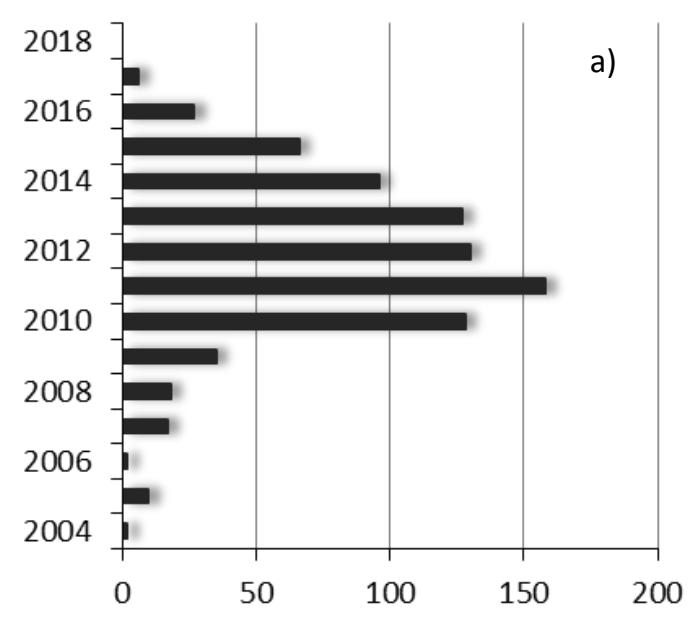

Número de contratos protocolados

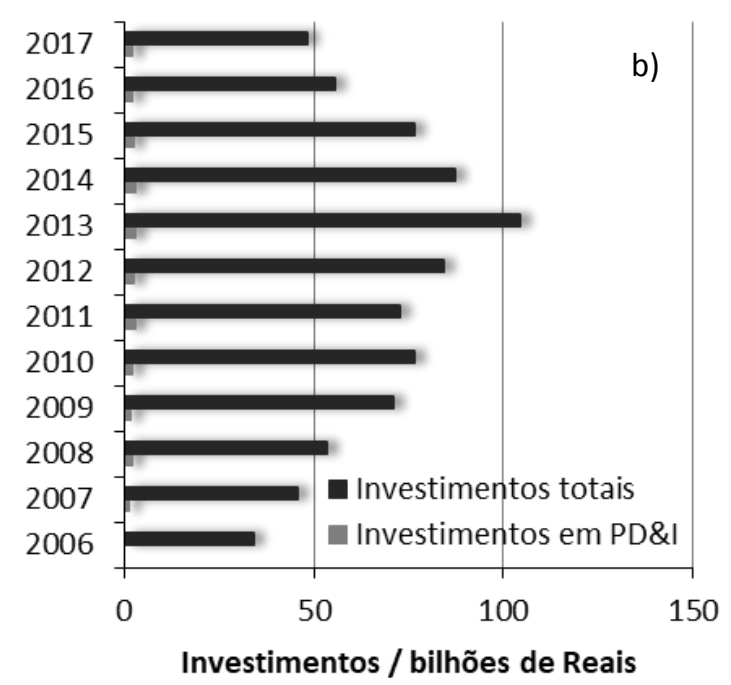

Investimentos / bilhões de Reais

Fontes: a) elaborado pelas autoras, a partir da base de dados de contratos do INPI (INSTITUTO..., 2018a). b) elaborado pelas autoras, a partir de dados disponibilizados nos Relatórios Anuais da Petrobras dos

\footnotetext{
${ }^{1}$ Acrônimo de Lucro Antes dos Juros, Impostos sobre Renda incluindo Contribuição Social sobre o Lucro Líquido, Depreciação e Amortização. É o resultado líquido do período, acrescido dos tributos sobre o lucro, das despesas financeiras líquidas das receitas financeiras e das depreciações, amortizações e exaustões (COMISSÃO..., 2012).
} 
anos entre 2006 e 2017, disponíveis em http://www.investidorpetrobras.com.br/pt/relatoriosanuais/relato-integrado/relatorio-anual. Acesso em: 01 jul. 18.

Considerando-se ainda o gráfico da Figura $1 b$, constata-se que houve uma diminuição de 54\% no montante de investimentos totais realizados pela Petrobras entre 2013 e 2017; no entanto, a diminuição no montante de investimentos em PD\&l foi de $25 \%$, no mesmo período. A partir dessa informação, pode-se inferir que nem todo financiamento da contratação internacional de tecnologia é proveniente dos investimentos em PD\&l realizados pela Petrobras.

\subsection{Partes contratantes}

As organizações estrangeiras contratadas pela Petrobras localizam-se principalmente na Europa $(55,3 \%)$ e na América do Norte $(39,0 \%)$, conforme pode ser observado no mapa da Figura 2, sendo que 340 organizações distintas foram contratadas para a transferência de conhecimento.

As organizações contratadas podem ser classificadas em empresas, universidades e institutos de PD\&l (público ou privado). Não há correlação direta entre a natureza da organização contratada e a modalidade do contrato, sendo que atividades de PD\&l foram contratadas nos três tipos de organizações (o mesmo pode ser afirmado em relação a atividades de prestação de serviços tecnológicos). Em estudo anterior, Spiandorello e Hoffmann (2017, p. 37) identificaram que a Petrobras contratou com 44 universidades estrangeiras no período entre 2007 e 2017.

Figura 2 - País ou região geográfica das 340 organizações estrangeiras contratadas (cedentes contratuais) no período entre 2004 e 2018. Os países destacados são os principais produtores mundiais de petróleo com os quais a Petrobras contrata organizações das respectivas nacionalidades

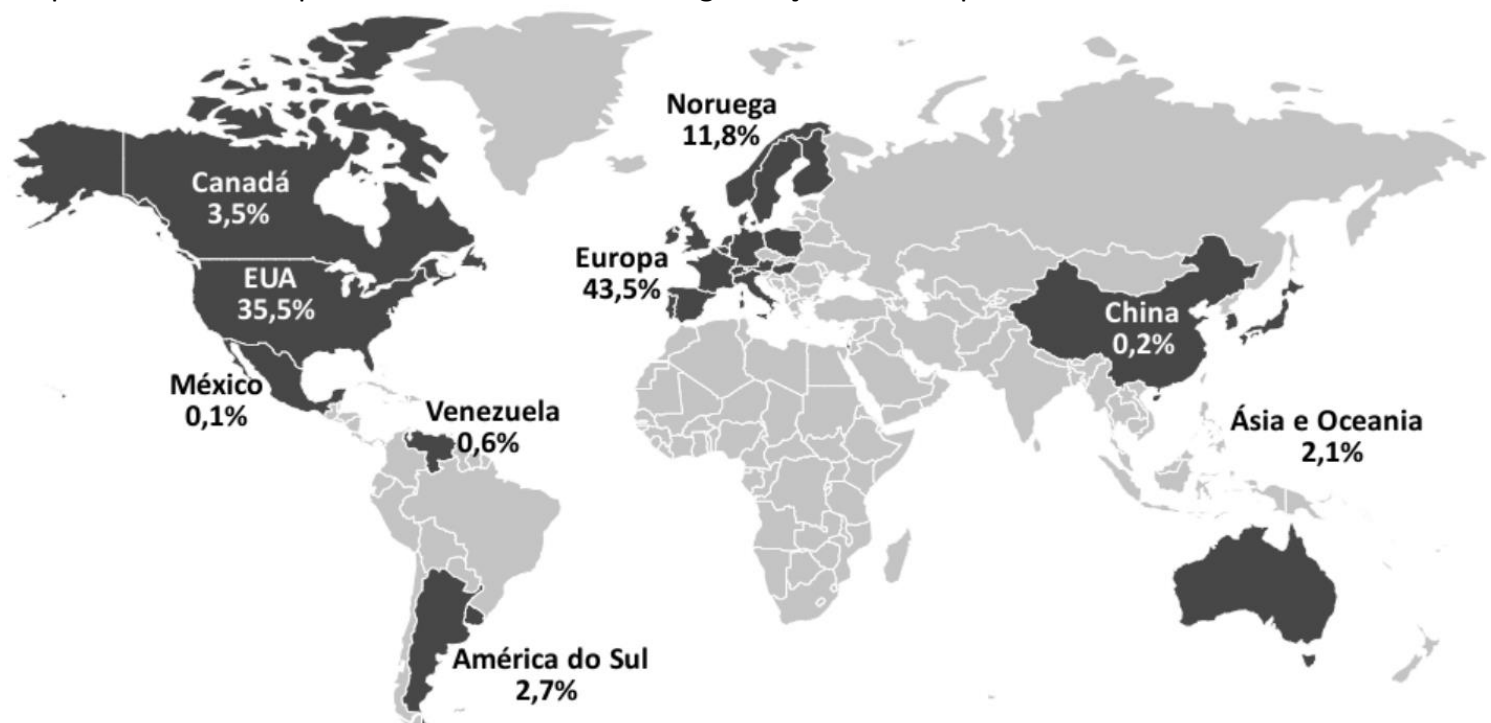

Fonte: elaborado pelas autoras, a partir da base de dados de contratos do INPI (INSTITUTO..., 2018a) e do uso do software Pixel Map Generator (amCharts), disponível em: http://pixelmap.amcharts.com/

Na Tabela 1 encontram-se nomeadas as organizações que têm o maior número de contratos internacionais de tecnologia firmados com a Petrobras, ou seja, maior frequência de contratação, bem como as que têm os contratos mais vultosos. Todos os contratos analisados têm a companhia Petróleo Brasileiro S. A. como contratante ou anuente.

Perspectivas em Gestão \& Conhecimento, João Pessoa, v. 8, número especial, p. 21-38, out. 2018. 
As organizações que são mais frequentemente contratadas caracterizam-se por serem grupos de empresas globais e institutos de pesquisa, públicos e privados. São fornecedoras de tecnologias necessárias ao design, operação e controle das plantas, por exemplo, podendo ser caracterizadas neste contexto como soft technologies.

Tabela 1 - Principais organizações contratadas pela Petrobras, considerando-se frequência e valor contratual

\begin{tabular}{|c|c|c|c|c|}
\hline $\begin{array}{l}\text { Cedente } \\
\text { contratual }\end{array}$ & País sede & Natureza e atividade principal & $\begin{array}{l}\text { Número de } \\
\text { contratos } \\
\text { protocolados }\end{array}$ & $\begin{array}{l}\text { Valor total } \\
\text { contratado }^{1}\end{array}$ \\
\hline \multicolumn{5}{|c|}{ Organizações mais frequentemente contratadas pela Petrobras - soft technologies } \\
\hline DNV GL & Noruega & $\begin{array}{l}\text { Grupo global de empresas. } \\
\text { Desenvolvimento de normas técnicas, } \\
\text { boas práticas operacionais, seguradora } \\
\text { marítima, gestão de risco, consultoria } \\
\text { técnica etc. }\end{array}$ & 51 & $R \$ 26.647 .973,89$ \\
\hline $\begin{array}{l}\text { IFP Énergies } \\
\text { Nouvelles - IFPEN }\end{array}$ & França & $\begin{array}{l}\text { Instituto público de PD\&I em energia, } \\
\text { transporte e meio ambiente }\end{array}$ & 31 & $\mathrm{R} \$ 14.611 .463,84$ \\
\hline TWI & $\begin{array}{l}\text { Reino } \\
\text { Unido }\end{array}$ & $\begin{array}{l}\text { Grupo de empresas. Instituto privado } \\
\text { de PD\&I especializado em soldagem. }\end{array}$ & 30 & $\mathrm{R} \$ 14.154 .976,88$ \\
\hline Axens S. A. & França & $\begin{array}{l}\text { Empresa startup do IFP Énergies } \\
\text { Nouvelles. Fornecedor de tecnologias } \\
\text { para indústria de óleo e gás, } \\
\text { particularmente catalisadores, serviços } \\
\text { e equipamentos. }\end{array}$ & 28 & $\mathrm{R} \$ 57.143 .064,86^{2}$ \\
\hline UOP Llc & $\begin{array}{l}\text { Estados } \\
\text { Unidos da } \\
\text { América }\end{array}$ & $\begin{array}{l}\text { Empresa do grupo Honeywell. } \\
\text { Fornecedora de tecnologia para a } \\
\text { indústria de óleo e gás }\end{array}$ & 22 & $\mathrm{R} \$ 1.110 .720 .853,02^{3}$ \\
\hline $\begin{array}{l}\text { Jacobs Nederland } \\
\text { BV }\end{array}$ & $\begin{array}{l}\text { Estados } \\
\text { Unidos da } \\
\text { América } \\
\end{array}$ & $\begin{array}{l}\text { Grupo global de empresas. Serviços de } \\
\text { engenharia para indústria de óleo e gás }\end{array}$ & 19 & $\mathrm{R} \$ 15.237 .902,28^{4}$ \\
\hline \multicolumn{5}{|c|}{ Organizações com contratos mais vultosos firmados com a Petrobras - hard technologies } \\
\hline UOP Llc & $\begin{array}{l}\text { Estados } \\
\text { Unidos da } \\
\text { América }\end{array}$ & $\begin{array}{l}\text { Empresa do grupo Honeywell. } \\
\text { Fornecedora de tecnologia para a } \\
\text { indústria de óleo e gás. }\end{array}$ & 22 & $\mathrm{R} \$ 1.110 .720 .853,02^{3}$ \\
\hline $\begin{array}{l}\text { Southern } \\
\text { Schlumberger S/A }\end{array}$ & Uruguai & $\begin{array}{l}\text { Empresa do grupo Schlumberger. Líder } \\
\text { mundial no fornecimento de tecnologia } \\
\text { para caracterização de reservatórios, } \\
\text { perfuração, produção e processamento } \\
\text { na indústria de óleo e gás. }\end{array}$ & 10 & $\mathrm{R} \$ 592.561 .067,33$ \\
\hline $\begin{array}{l}\text { Forster Wheeler } \\
\text { Energy Limited }\end{array}$ & Suíça & $\begin{array}{l}\text { Empresa do grupo Wood. Líder global } \\
\text { em projetos de engenharia e serviços } \\
\text { técnico para setores industriais e de } \\
\text { energia. }\end{array}$ & 2 & $R \$ 413.514 .646,81$ \\
\hline $\begin{array}{l}\text { Toyo Engineering } \\
\text { Corporation }\end{array}$ & Japão & $\begin{array}{l}\text { Empresa transnacional. Atua no } \\
\text { segmento de engenharia e construção } \\
\text { de instalações industriais. }\end{array}$ & 2 & $R \$ 242.178 .061,69$ \\
\hline $\begin{array}{l}\text { Halliburton } \\
\text { Energy Services } \\
\text { Inc. }\end{array}$ & $\begin{array}{l}\text { Estados } \\
\text { Unidos da } \\
\text { América }\end{array}$ & $\begin{array}{l}\text { Empresa do grupo Halliburton. } \\
\text { Fornecedora de produtos e serviços } \\
\text { para indústria de óleo e gás. }\end{array}$ & 15 & $R \$ 226.076 .386,99$ \\
\hline $\begin{array}{l}\text { Baker Hughes B. } \\
\text { V. e Baker Hughes } \\
\text { Oilfield } \\
\text { Operations, Inc. }\end{array}$ & $\begin{array}{l}\text { Estados } \\
\text { Unidos da } \\
\text { América }\end{array}$ & $\begin{array}{l}\text { Empresas do grupo GE. Fornecedoras } \\
\text { de produtos, serviços e soluções } \\
\text { digitais integradas para campos de } \\
\text { petróleo. }\end{array}$ & 7 & $\mathrm{R} \$ 163.284 .671,02^{5}$ \\
\hline
\end{tabular}

Perspectivas em Gestão \& Conhecimento, João Pessoa, v. 8, número especial, p. 21-38, out. 2018. 
Notas: 1) Os valores contratuais originais foram convertidos para a moeda nacional (taxa de conversão do dia do protocolo do pedido de registro/averbação) e atualizados por meio do índice IGP-M de maio de 2018. 2) Há cinco contratos sem publicação de valores. 3) Há três contratos sem publicação de valores. 4) Há um contrato sem publicação de valor. 5) Há três contratos sem publicação de valores.

A empresa DNV GL conta com a maior frequência de contratações por parte da Petrobras, sendo que todos os contratos são da modalidade de Serviços de Assistência Técnica, podendo ser caracterizados como PD\&I, prestação de serviços ou consultoria. Dantas e Bell (2009), ao estudarem o desenvolvimento de redes de conhecimento da Petrobras, identificaram que houve uma evolução no tipo de conhecimento transferido pela DNV GL: não mais apenas voltada para a certificação de plataformas (prestação de serviços), mas para o desenvolvimento de processos para o projeto de plataformas (consultoria).

O IFPEN é a segunda organização mais frequentemente contratada pela Petrobras, sendo que o valor médio de seus contratos é de $\mathrm{R} \$ 471.337,54$. Já os contratos firmados com a empresa Axens S. A., uma startup criada pelo IFPEN no ano de 2001, têm valor médio de $\mathrm{R} \$ 2.040 .826,74$. Todos os contratos tipo FT foram firmados com a Axens S. A., enquanto que os contratos de PD\&l foram firmados com o IFPEN; ambas organizações têm contratos de prestação de serviços firmados com a Petrobras. Os valores e os tipos de contratos demonstram a importância do desenvolvimento industrial - realizado pela Axens S. A. necessário para agregar valor ao conhecimento científico e tecnológico desenvolvido nos institutos de pesquisa, no caso o IFPEN. Atualmente, $50 \%$ do financiamento das atividades do IFPEN é decorrente do setor privado, particularmente das receitas advindas de suas treze startups, como a Axens S. A. (IFP..., 2018).

Quando se analisam as empresas contratadas pelos maiores valores, verifica-se tratar de produtos e serviços voltados à infraestrutura e à operação de refinarias e poços de petróleo (hard technologies, contextualmente), o que explica os valores elevados das contratações e a baixa frequência.

A empresa UOP LIc é a companhia com maiores valores contratados, sendo também uma das que é mais frequentemente contratada. Isso se deve à natureza da empresa: tendo sido constituída em 1914, já com o objetivo de comercializar conhecimento voltado ao refino de petróleo, veio diversificando suas atividades, sendo capaz também de executar projetos de infraestrutura.

\subsection{Modalidades e objetos contratuais}

Considerando-se as modalidades contratuais reconhecidas pelo INPI, conforme descrito no referencial teórico, os contratos internacionais estabelecidos pela Petrobras classificam-se em EP, EP/FT, FT, FT/SAT e SAT. Os contratos de serviços de assistência técnica (SAT) podem ser classificados, quanto a seu objeto, em consultoria (CST), prestação de serviços tecnológicos (PST) e parcerias para pesquisa e desenvolvimento (PD\&I), sendo que uma visão geral das modalidades e objetos contratados pela Petrobras encontra-se demonstrada na Tabela 2. O fato de $94 \%$ das contratações realizadas pela Petrobras serem em serviços de assistência técnica (SAT) é um indicativo de que esta modalidade é preferível em relação ao licenciamento de patentes (EP) ou de know-how (FT), tendo em vista que ao final daquelas atividades contratadas os resultados técnicos do projeto, ou seja, a propriedade intelectual, pertencem à contratante.

Perspectivas em Gestão \& Conhecimento, João Pessoa, v. 8, número especial, p. 21-38, out. 2018. 
Tabela 2 - Modalidades e objetos dos contratos internacionais de transferência de tecnologia

\begin{tabular}{l|c|c|c|c|c|c|c}
\hline Contrato-tipo & EP & EP/FT & FT & FT/SAT & \multicolumn{3}{c}{ SAT } \\
\hline Objeto contratual & - & - & - & - & CST & PST & PD\&I \\
\hline Ocorrência (\%) & 0,5 & 1,0 & 4,0 & 0,5 & 16,0 & 43,4 & 34,6 \\
\hline
\end{tabular}

Fonte: elaborado pelas autoras, a partir da base de dados de contratos do INPI (INSTITUTO..., 2018a)

De acordo com a UNCTAD (UNITED..., 2014, p. 15-19), existem quatro canais para a transferência internacional de tecnologia e conhecimento: comércio, licenciamento, investimento estrangeiro direto e movimentação de recursos humanos. Sob essa ótica, os dados coletados indicam que as contratações realizadas pela Petrobras podem ser classificadas como comércio de serviços (SAT) - apesar da classificação adotada se referir unicamente ao comércio de bens - licenciamento (EP e FT) e movimentação de recursos humanos (CST).

\subsection{Valores e prazos de vigência contratuais}

Analisando-se os prazos declarados no primeiro certificado emitido para cada registro, construiu-se a curva de distribuição de vigência contratual apresentada na Figura 3. Em sua maioria (cerca de 60\%), as parcerias internacionais formadas têm prazo inferior a 24 meses; cerca de $88 \%$ têm prazo inferior a 48 meses. Os contratos com valores de até R\$ 5 milhões constituem $89 \%$ das contratações internacionais realizadas pela Petrobras (Figura 4).

Figura 3 - Distribuição dos prazos de vigência dos contratos de transferência de tecnologia (primeira averbação/registro). Não se encontram contabilizados aditivos contratuais com extensão do prazo de vigência

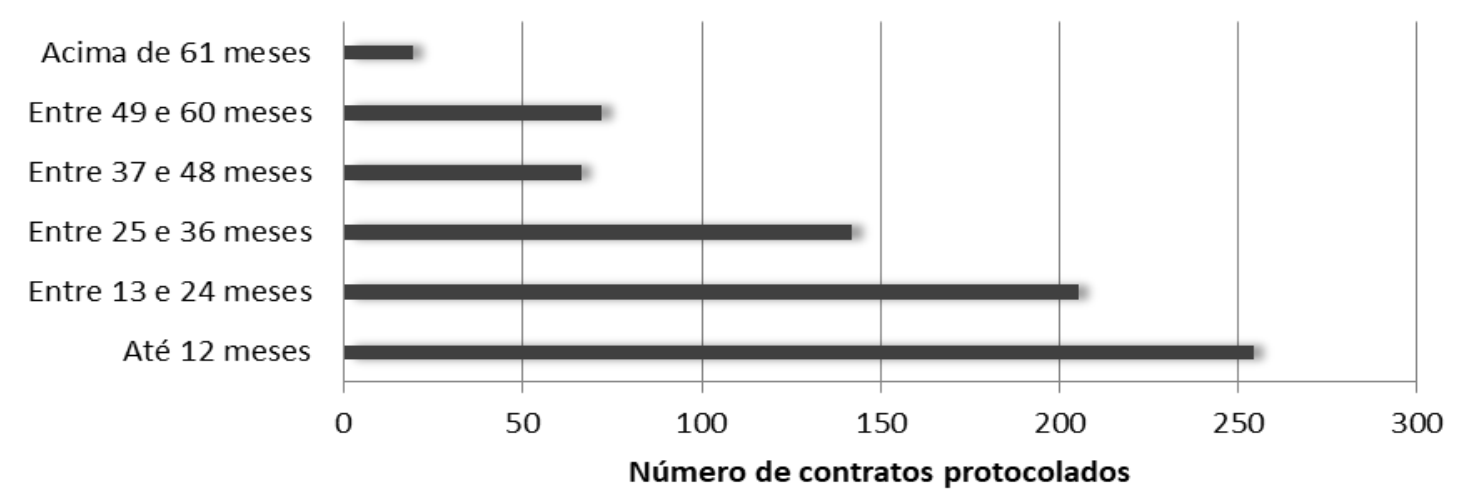

Fonte: Elaborado pelas autoras, a partir da base de dados de contratos do INPI (INSTITUTO..., 2018a) 
Figura 4 - Distribuição dos valores contratuais do primeiro registro/averbação. Não se encontram contabilizados aditivos contratuais com extensão do prazo de vigência

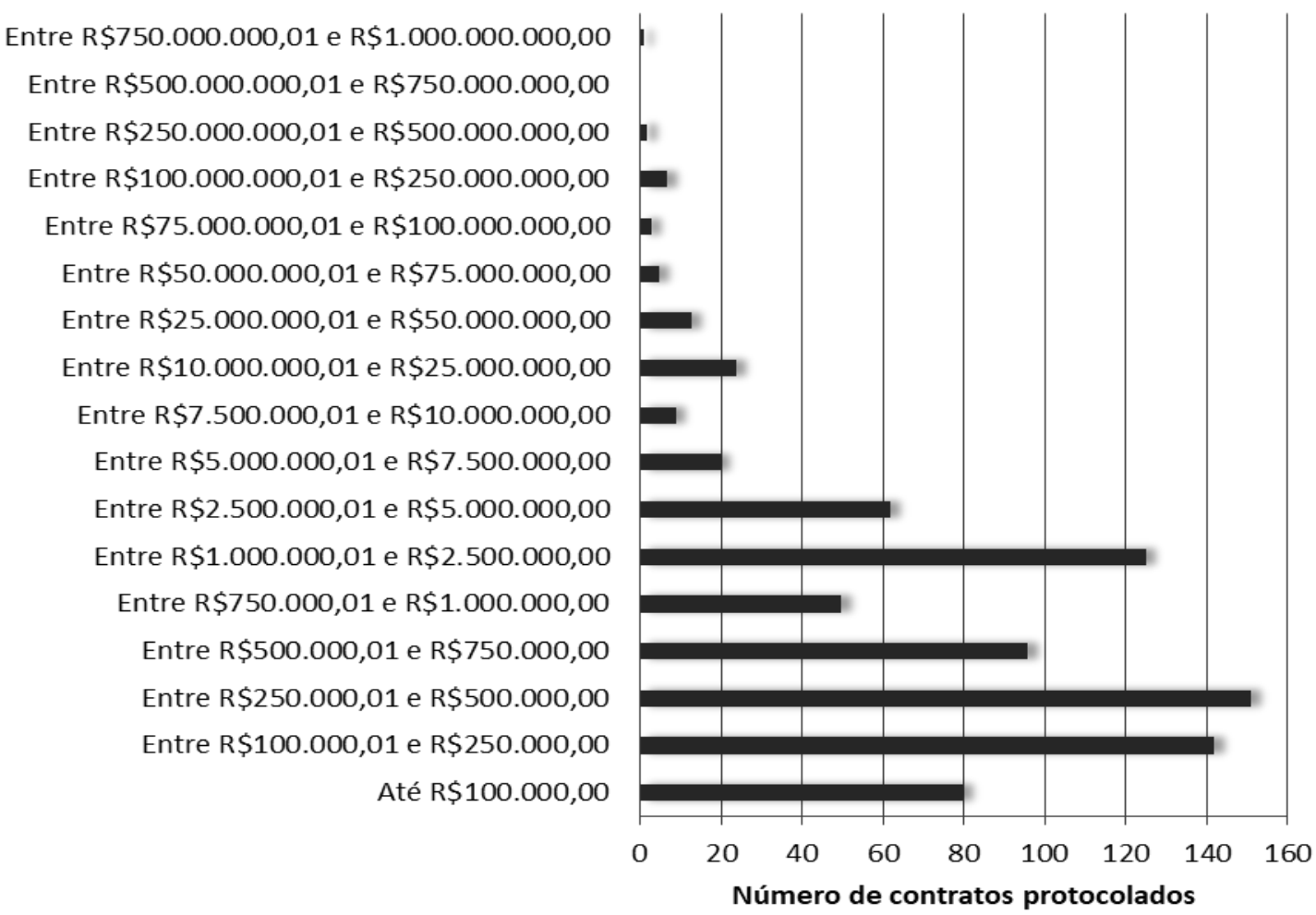

Fonte: Elaborado pelas autoras, a partir da base de dados de contratos do INPI (INSTITUTO..., 2018a). Nota: Os valores contratuais originais foram convertidos para a moeda nacional (taxa de conversão do dia do protocolo do pedido de registro/averbação) e atualizados por meio do índice IGP-M de maio de 2018

Ao relacionarem-se os objetos contratuais em função da vigência e do valor médio do instrumento (Tabela 3), constata-se que os contratos SAT têm vigência média inferior a das demais modalidades. Isso é esperado já que serviços tecnológicos, mesmo os que envolvem parcerias PD\&l, têm caráter mais efêmero que os de licenciamento (EP, FT e suas combinações), sendo que não há transmissão de direitos sobre propriedade intelectual. Os maiores valores contratuais são referentes às modalidades EP e EP/FT, nas quais há o licenciamento de direitos de propriedade intelectual, no caso patentes.

Tabela 3 - Modalidades, objetos e valores médios dos contratos internacionais de transferência de tecnologia firmados pela Petrobras

\begin{tabular}{l|c|c|c|c|c|c|c}
\hline \multicolumn{1}{c}{ Contrato-tipo } & EP & EP/FT & FT & FT/SAT & \multicolumn{3}{c}{ SAT } \\
\hline Objeto contratual & - & - & - & - & CST & PST & PD\&I \\
\hline $\begin{array}{l}\text { Vigência média } \\
\text { (meses) }\end{array}$ & 66 & 40 & 53 & 56 & 20 & 24 & 25 \\
\hline $\begin{array}{l}\text { Valor médio } \\
\text { (milhões de Reais) }\end{array}$ & 63,25 & 18,52 & 7,54 & 6,64 & 1,94 & 13,54 & 1,35 \\
\hline
\end{tabular}

Fonte: elaborado pelas autoras, a partir da base de dados de contratos do INPI (INSTITUTO..., 2018a).

Nota: Os valores contratuais originais foram convertidos para a moeda nacional (taxa de conversão do dia do protocolo do pedido de registro/averbação) e atualizados por meio do índice IGP-M de maio de 2018.

Perspectivas em Gestão \& Conhecimento, João Pessoa, v. 8, número especial, p. 21-38, out. 2018. 
Vale ressaltar que não foram identificados contratos tipo EP ou FT que sejam o licenciamento de patentes ou de know-how desenvolvidos pelo estabelecimento de parcerias SAT, particularmente PD\&I, ou seja, há indicativos que nos contratos SAT há cláusulas que determinam que a propriedade dos resultados seja integralmente da parte contratante, no caso a Petrobras, não havendo possibilidade de compartilhamento dos resultados.

\subsection{Contratos de Exploração de Patentes (EP) e de Fornecimento de Tecnologia (FT)}

Os contratos-tipo EP e FT agregam informações particularmente relevantes quando se deseja compreender as alianças envolvidas na transferência internacional de tecnologia; podese obter as primeiras informações acerca das empresas que direta - ou indiretamente integram as parcerias para licenciamento de patentes e know-how.

$\mathrm{Na}$ Tabela 4 encontram-se listadas as empresas contratadas pela Petrobras para o licenciamento de patentes (EP) e know-how (FT). Algumas são as principais parceiras de fornecimento de tecnologia, como anteriormente identificado na Tabela 1.

Tabela 4 - Contratos internacionais de transferência de tecnologia para fornecimento de tecnologia e exploração de patentes: correlação com as patentes envolvidas nas transações

\begin{tabular}{|c|c|c|c|c|}
\hline Cedente contratual & $\begin{array}{l}\text { Patente } \\
\text { brasileira }\end{array}$ & Titular da patente & $\begin{array}{c}\text { Data do } \\
\text { depósito da } \\
\text { patente }\end{array}$ & $\begin{array}{c}\text { Data de } \\
\text { protocolo } \\
\text { do contrato }\end{array}$ \\
\hline $\begin{array}{l}\text { Transocean Offshore } \\
\text { Deepwater Drilling } \\
\text { Inc. }\end{array}$ & $\begin{array}{l}\text { PI9706592-7 } \\
\text { PI9715094-0 }\end{array}$ & $\begin{array}{l}\text { Transocean Offshore Deepwater } \\
\text { Drilling Inc. (US) } \\
\text { Transocean Offshore Deepwater } \\
\text { Drilling Inc. (US) }\end{array}$ & $\begin{array}{l}27 / 01 / 1997 \\
27 / 01 / 1997\end{array}$ & $\begin{array}{l}01 / 02 / 2017 \\
\text { (EP) }\end{array}$ \\
\hline $\begin{array}{l}\text { Technip S\&W } \\
\text { International, Inc. }\end{array}$ & $\begin{array}{l}\text { PI0110721-6 } \\
\text { PI0312830-0 } \\
\text { PI0312894-6 } \\
\end{array}$ & $\begin{array}{l}\text { BASF Aktiengesellschaft (DE) } \\
\text { BASF Aktiengesellschaft (DE) } \\
\text { BASF Aktiengesellschaft (DE) }\end{array}$ & $\begin{array}{l}09 / 05 / 2001 \\
22 / 07 / 2003 \\
23 / 07 / 2003 \\
\end{array}$ & $\begin{array}{c}27 / 09 / 2013 \\
\text { (EP/FT) }\end{array}$ \\
\hline $\begin{array}{l}\text { Foster Wheeler } \\
\text { Energy Limited }\end{array}$ & $\begin{array}{l}\text { PI0816071-6 } \\
\text { PI0819277-4 }\end{array}$ & $\begin{array}{l}\text { Lurgi Gmbh (DE) } \\
\text { Saipem S.P.A. (IT) }\end{array}$ & $\begin{array}{l}18 / 08 / 2008 \\
14 / 11 / 2008\end{array}$ & $\begin{array}{c}13 / 02 / 2013 \\
(E P / F T)\end{array}$ \\
\hline UOP Llc & PI9606026-3 & UOP, Inc. (US) & $16 / 12 / 1996$ & $\begin{array}{c}19 / 08 / 2014 \\
\text { (EP/FT) } \\
05 / 11 / 2012 \\
\text { (EP/FT) } \\
23 / 03 / 2012 \\
\text { (EP/FT) } \\
03 / 10 / 2011 \\
\text { (EP/FT) }\end{array}$ \\
\hline Jacobs Nederland BV & PI9610543-7 & Jacobs Nederland BV (NL) & 16/09/1996 & $\begin{array}{l}07 / 02 / 2011 \\
\text { (EP) } \\
14 / 07 / 2009 \\
\text { (EP) }\end{array}$ \\
\hline $\begin{array}{l}\text { Stone \& Webster } \\
\text { International, Inc. }\end{array}$ & $\begin{array}{l}\text { PI0110721-6 } \\
\text { PI0115431-1 } \\
\text { PI0312830-0 } \\
\text { PI0312894-6 }\end{array}$ & $\begin{array}{l}\text { BASF Aktiengesellschaft (DE) } \\
\text { BASF Aktiengesellschaft (DE) } \\
\text { BASF Aktiengesellschaft (DE) } \\
\text { BASF Aktiengesellschaft (DE) }\end{array}$ & $\begin{array}{l}09 / 05 / 2001 \\
15 / 11 / 2001 \\
22 / 07 / 2003 \\
23 / 07 / 2003\end{array}$ & $\begin{array}{c}01 / 08 / 2008 \\
\text { (EP/FT) }\end{array}$ \\
\hline Axens S.A. & $\begin{array}{l}\text { PI9501828-0 } \\
\text { PI9502877-3 } \\
\text { PI9607590-2 } \\
\text { PI9703955-1 } \\
\text { PI9801520-6 } \\
\text { PI9909696-0 } \\
\text { PI0006676-1 } \\
\text { PI0002861-4 } \\
\text { PI9913291-5 }\end{array}$ & $\begin{array}{l}\text { Institut Français du Petrole (FR) } \\
\text { Institut Français du Petrole (FR) } \\
\text { Mobil Oil Corporation (US) } \\
\text { Institut Français du Petrole (FR) } \\
\text { Institut Français du Petrole (FR) } \\
\text { Mobil Oil Corporation (US) } \\
\text { Institut Français du Petrole (FR) } \\
\text { Institut Français du Petrole (FR) } \\
\text { Institut Français du Petrole (FR) }\end{array}$ & $\begin{array}{l}27 / 04 / 1995 \\
21 / 06 / 1995 \\
09 / 02 / 1996 \\
11 / 07 / 1997 \\
29 / 04 / 1998 \\
12 / 04 / 1999 \\
26 / 05 / 2000 \\
23 / 06 / 2000 \\
30 / 08 / 1999\end{array}$ & $\begin{array}{c}21 / 02 / 2008 \\
\text { (EP) }\end{array}$ \\
\hline
\end{tabular}

Perspectivas em Gestão \& Conhecimento, João Pessoa, v. 8, número especial, p. 21-38, out. 2018. 


\begin{tabular}{l|c|l|l|l}
\hline & PI9913370-9 & Institut Français du Petrole (FR) & $30 / 08 / 1999$ & \\
& PI0208050-8 & Institut Français du Petrole (FR) & $29 / 01 / 2002$ & \\
\hline
\end{tabular}

Fonte: elaborado pelas autoras, a partir das bases de dados de contratos (INSTITUTO..., 2018a) e de patentes (INSTITUTO..., 2018b) do INPI, e da base de dados Espacenet (EUROPEAN..., 2018)

Analisando-se as titulares das patentes licenciadas para a Petrobras, identificam-se casos como da Technip S\&W, Stone \& Webster e Axens S. A., nos quais o que ocorre são sublicenciamentos de patentes, tendo em vista que os titulares são BASF Aktiengesellschaft, Institut Français du Petrole (atual IFPEN) e Mobil Oil Corporation, respectivamente. As demais empresas cedentes são titulares das patentes licenciadas. Infere-se que a Technip S\&W é sucessora da Stone \& Webster.

Dos dados constantes da Tabela 4, depreende-se que à exceção dos contratos firmados com as empresas UOP LIc., Transocean Offshore Deepwater Drilling Inc. e Jacobs Nederland BV, os demais contratos são estabelecidos com empresas que são licenciadas pelas titulares originais das patentes, estabelecendo-se então contratos com cláusulas de sublicenciamento de uso das tecnologias. Particularmente no caso do contrato EP firmado com a Axens S. A., as áreas tecnológicas das patentes licenciadas são complementares entre si, conforme informações da Tabela 5.

Os valores pagos pela Petrobras para o licenciamento das patentes são evidenciados na Tabela 5 , juntamente com o prazo dos contratos e a área tecnológica a qual a matéria da patente é pertinente.

Tabela 5 - Prazo de vigência, valor e área tecnológica das patentes licenciadas ou sublicenciadas pela Petrobras

\begin{tabular}{|c|c|c|c|c|}
\hline $\begin{array}{l}\text { Cedente } \\
\text { contratual }\end{array}$ & $\begin{array}{l}\text { Patente } \\
\text { brasileira }\end{array}$ & Título da patente & $\begin{array}{c}\text { Prazo } \\
\text { contratual } \\
\text { (meses) }\end{array}$ & $\begin{array}{l}\text { Valor total } \\
\text { contratado }\end{array}$ \\
\hline \multirow{2}{*}{$\begin{array}{l}\text { Transocean } \\
\text { Offshore } \\
\text { Deepwater } \\
\text { Drilling Inc. }\end{array}$} & PI9706592-7 & $\begin{array}{l}\text { Exploração em alto mar com múltiplas } \\
\text { atividades e/ou método e aparelho de } \\
\text { perfuração em desenvolvimento }\end{array}$ & \multirow{2}{*}{2} & \multirow{2}{*}{$\mathrm{R} \$ 127.089 .299,59$} \\
\hline & PI9715094-0 & $\begin{array}{l}\text { Navio de perfuração e montagem de } \\
\text { perfuração de atividades múltiplas }\end{array}$ & & \\
\hline \multirow{3}{*}{$\begin{array}{l}\text { Technip S\&W } \\
\text { International, } \\
\text { Inc. }\end{array}$} & PI0110721-6 & $\begin{array}{l}\text { Processo para tratar uma fração } \mathrm{C} 4, \mathrm{e} \\
\text { dispositivo para realizar o mesmo }\end{array}$ & \multirow{3}{*}{13} & \multirow{3}{*}{$\mathrm{R} \$ 621.638,80$} \\
\hline & PI0312830-0 & $\begin{array}{l}\text { Processo contínuo para a separação de } \\
\text { uma fração C4 por destilação extrativa } \\
\text { com um solvente seletivo em uma coluna } \\
\text { de destilação extrativa }\end{array}$ & & \\
\hline & PI0312894-6 & $\begin{array}{l}\text { Processo contínuo para separação de uma } \\
\text { mistura de hidrocarbonetos obtidos de } \\
\text { uma fração C4 por meio de destilação } \\
\text { extrativa }\end{array}$ & & \\
\hline \multirow{2}{*}{$\begin{array}{l}\text { Foster } \\
\text { Wheeler } \\
\text { Energy } \\
\text { Limited }\end{array}$} & PI0816071-6 & $\begin{array}{l}\text { Processo e instalação para a produção de } \\
\text { metanol }\end{array}$ & \multirow{2}{*}{2} & \multirow{2}{*}{$\mathrm{R} \$ 4.543 .394,06$} \\
\hline & PI0819277-4 & $\begin{array}{l}\text { Processo de recuperação de amônia, e, } \\
\text { equipamento para realizar o processo }\end{array}$ & & \\
\hline UOP Llc & PI9606026-3 & $\begin{array}{l}\text { Aparelhos para desprender partículas de } \\
\text { um tubo de elevação e para o transporte }\end{array}$ & 54 & $\mathrm{R} \$ 120.731 .062,78$ \\
\hline
\end{tabular}

Perspectivas em Gestão \& Conhecimento, João Pessoa, v. 8, número especial, p. 21-38, out. 2018. 


\begin{tabular}{|c|c|c|c|c|}
\hline & & $\begin{array}{l}\text { de material particulado mais } \\
\text { especificamente apropriados para uso em }\end{array}$ & 36 & $R \$ 1.665 .072,28$ \\
\hline & & respectivo processo de reformação & 54 & $R \$ 1.564 .532,24$ \\
\hline & & & 48 & $\mathrm{R} \$ 455.084,93$ \\
\hline \multirow{2}{*}{$\begin{array}{l}\text { Jacobs } \\
\text { Nederland BV }\end{array}$} & \multirow{2}{*}{ PI9610543-7 } & \multirow{2}{*}{$\begin{array}{l}\text { Processo e aparelho adequado para } \\
\text { remover compostos de sulfeto de } \\
\text { hidrogênio de enxofre líquido }\end{array}$} & 70 & $\mathrm{R} \$ 162.730,14$ \\
\hline & & & 85 & $\begin{array}{l}\text { Valor contratual } \\
\text { não publicado }\end{array}$ \\
\hline \multirow{4}{*}{$\begin{array}{l}\text { Stone \& } \\
\text { Webster } \\
\text { International, } \\
\text { Inc. }\end{array}$} & PI0110721-6 & $\begin{array}{l}\text { Processo para tratar uma fração } \mathrm{C} 4, \mathrm{e}, \\
\text { dispositivo para realizar o mesmo }\end{array}$ & \multirow{4}{*}{32} & \multirow{4}{*}{$\mathrm{R} \$ 55.756,69$} \\
\hline & PI0115431-1 & $\begin{array}{l}\text { Processo para a obtenção, por meio de } \\
\text { destilação, de 1,3-butadieno puro a partir } \\
\text { de 1,3-butadieno bruto, e coluna de } \\
\text { parede divisória }\end{array}$ & & \\
\hline & PI0312830-0 & $\begin{array}{l}\text { Processo contínuo para a separação de } \\
\text { uma fração C4 por destilação extrativa } \\
\text { com um solvente seletivo em uma coluna } \\
\text { de destilação extrativa }\end{array}$ & & \\
\hline & PI0312894-6 & $\begin{array}{l}\text { Processo contínuo para separação de uma } \\
\text { mistura de hidrocarbonetos obtidos de } \\
\text { uma fração C4 por meio de destilação } \\
\text { extrativa }\end{array}$ & & \\
\hline \multirow{8}{*}{ Axens S. A. } & PI9501828-0 & $\begin{array}{l}\text { Processo de separação em camada móvel } \\
\text { simulada com vazão de reciclagem } \\
\text { constante }\end{array}$ & \multirow{8}{*}{147} & \multirow{8}{*}{$\begin{array}{l}\text { Valor contratual } \\
\text { não publicado }\end{array}$} \\
\hline & PI9502877-3 & $\begin{array}{l}\text { Processo de separação em camada móvel } \\
\text { simulada a partir de uma carga de pelo } \\
\text { menos dois constituintes }\end{array}$ & & \\
\hline & PI9607590-2 & Processamento de aromáticos pesados & & \\
\hline & PI9703955-1 & $\begin{array}{l}\text { Dispositivo de enxágue em uma unidade } \\
\text { de adsorção em leito móvel simulado e } \\
\text { sua utilização }\end{array}$ & & \\
\hline & PI9801520-6 & $\begin{array}{l}\text { Método para controlar com precisão um } \\
\text { processo de separação de constituintes de } \\
\text { uma mistura, em um sistema de separação } \\
\text { com camada móvel simulada }\end{array}$ & & \\
\hline & PI9909696-0 & $\begin{array}{l}\text { Processo para isomerização de uma } \\
\text { corrente compreendendo etilbenzeno e } \\
\text { xileno }\end{array}$ & & \\
\hline & PI0006676-1 & $\begin{array}{l}\text { Dispositivo que permite separar pelo } \\
\text { menos um composto a partir de uma } \\
\text { mistura ou de um corpo por adsorção e } \\
\text { processo de injeção de fluido desviado em } \\
\text { um processo de separação por leito móvel } \\
\text { simulado }\end{array}$ & & \\
\hline & PI0002861-4 & $\begin{array}{l}\text { Processo de hidrotratamento de um } \\
\text { destilado médio e duas zonas sucessivas } \\
\text { compreendendo uma zona intermediária } \\
\text { de remoção do efluente da primeira zina } \\
\text { com condensação dos produtos pesados } \\
\text { que saem na cabeça do removedor }\end{array}$ & & \\
\hline
\end{tabular}

Perspectivas em Gestão \& Conhecimento, João Pessoa, v. 8, número especial, p. 21-38, out. 2018. 


\begin{tabular}{|l|l|}
\hline PI9913291-5 & $\begin{array}{l}\text { Distribuidor-misturador-extrator de fluidos } \\
\text { e processo associado }\end{array}$ \\
\hline PI9913370-9 & $\begin{array}{l}\text { Sistema de coleta-distribuição de fluido e } \\
\text { seu processo }\end{array}$ \\
\hline PI0208050-8 & $\begin{array}{l}\text { Processo de produção de gasolina } \\
\text { dessulfurada, a partir de um corte } \\
\text { gasolina, contendo a gasolina de } \\
\text { conversão }\end{array}$ \\
\hline
\end{tabular}

Fontes: Elaborado pelas autoras, a partir da base de dados de contratos do INPI (INSTITUTO..., 2018a)

Notas: 1) Os valores contratuais originais foram convertidos para a moeda nacional (taxa de conversão do dia do protocolo do pedido de registro/averbação) e atualizados por meio do índice IGP-M de maio de 2018.

No caso da patente PI96060626-3, a estratégia adotada pela empresa UOP Llc foi firmar diversos contratos de licenciamentos não exclusivos, cada um destinado a uma refinaria da Petrobras (v. g., Repar, Revap, Replan e PRBC).

A patente PI9706592-7, de titularidade da Transocean Offshore Deepwater Drilling Inc., foi extinta em 27/01/2017 (INSTITUTO..., 2018b), sendo que o contrato EP foi protocolado em 01/02/2017 (Tabela 4), ou seja, após sua extinção; a patente PI9715094-0 continua vigente. Considerando-se que o prazo contratual para exploração de patentes era apenas de dois meses - bem como o valor elevado do contrato (Tabela 5 ) - pode-se inferir que o real valor da tecnologia não estava protegido pelos direitos de propriedade intelectual, mas nos equipamentos descritos nas patentes, que tratam de navios para perfuração de poços de petróleo em alto mar (INSTITUTO..., 2018b). Os casos das patentes contratadas com as empresas Technip S\&W International, Inc., Foster Wheeler Energy Limited e Jacobs Nederland BV também têm valor associado a equipamentos descritos nos documentos patentários.

\section{CONCLUSÕES}

Entende-se que as relações contratuais tecnológicas estabelecidas entre empresas trazem em seu bojo uma quantidade de informações relevantes, podendo ser utilizadas na formação da inteligência competitiva de organizações. As informações constantes dos extratos contratuais analisados permitiram o mapeamento das alianças estratégicas internacionais estabelecidas pela Petrobras, identificando-se as organizações contratadas para realização de atividades voltadas à transferência de conhecimento, tais como licenciamento de patentes e de know-how, serviços tecnológicos, consultoria e parcerias PD\&I. Outras características das parcerias, como valores contratuais e prazo, também são possíveis de serem identificadas.

Ao cruzarem-se os dados contratuais com os documentos patentários, verificaram-se informações pertinentes à Cadeia Global de Valor em inovação, sendo que foi possível a identificação de organizações que originariamente realizam atividades de PD\&l e têm o licenciamento de patentes e know-how como seu modelo de negócios, como é o caso da empresa alemã BASF Aktiengesellschaft e do instituto público francês IFP Energies Nouvelles IFPEN. Também foi possível a identificação das áreas tecnológicas contratadas pela Petrobras para complementar seu capital intelectual. Corroborou-se o estudo de Perrons (2014), que ao estudar atividades de pesquisa e desenvolvimento (P\&D) na indústria de óleo de gás, constatou que as empresas de serviços são a principal fonte de inovações para essa indústria.

Com este trabalho foi possível demonstrar a viabilidade do mapeamento detalhado das alianças estratégicas internacionais estabelecidas por empresas brasileiras utilizando-se as informações disponibilizadas pública e gratuitamente. Devido ao fato da Petrobras ser uma 
empresa pública, muitos desses dados contratuais encontram-se também disponibilizados no "Portal da Transparência" da companhia (http://transparencia.petrobras.com.br/licitacoescontratos/contratos); mas isso não ocorre para empresas de natureza privada, que não têm obrigação de realizar contratações públicas. Este é um valor imediato que se pode extrair das informações em questão. Valores mediatos que podem ser alcançados com a análise detalhada e cruzamento das informações: mapeamento das redes de conhecimento construídas para desenvolvimento de novas tecnologias, estimativas de custos internacionais e prazos envolvidos em projetos específicos, modelos de transferência de tecnologia adotados para projetos de PD\&l etc. Também é possível acompanhar a evolução temporal da inserção da empresa nas CGV e das tecnologias fornecidas para a execução de seus projetos. Todas estas informações têm valor estratégico, corroborando a importância da formação de programas perenes de Inteligência Competitiva para a tomada de decisão em organizações.

\section{AGRADECIMENTOS}

O presente trabalho foi realizado com apoio da Coordenação de Aperfeiçoamento de Pessoal de Nível Superior (CAPES), Brasil. Código de Financiamento 001.

\section{REFERÊNCIAS}

AYRES, M. Petrobras cria programa para acelerar desinvestimentos. Revista Exame. 2012. Disponível em: https://exame.abril.com.br/negocios/petrobras-cria-programa-para-acelerardesinvestimentos/. Acesso em: 29 jun. 2018.

CALOF, J. Competitive intelligence practices of European firms. Technology Analysis \& Strategic Management, v. 30, n. 6, 2018, p. 658-671.

CHESBROUGH, H. W. Open Innovation: Researching a new paradigm. Oxford: Oxford University Press, 392 p., 2006.

COMISSÃO DE VALORES MOBILIÁRIOS - CVM. Instrução CVM no 527, de 4 de outubro de 2012. Dispõe sobre a divulgação voluntária de informações de natureza não contábil denominadas LAJIDA L LAJIR. Disponível em: http://www.cvm.gov.br/export/sites/cvm/legislacao/instrucoes/anexos/500/inst527.pdf.

Acesso em: 29 jun. 2018.

CRISCUOLO, C.; TIMMIS, J. The changing structure of GVCS: are central hubs key for productivity? In: Conference of the Global Forum on Productivity. 2017, Budapest.

CURTIS, J. Behind enemy lines. Marketing, 2001. p. 28-29.

DANTAS, E.; BELL, M. Latecomer firms and the emergence and development of knowledge networks: The case of Petrobras in Brazil. Research Policy, n. 38, 2009, p. 829-844.

DUARTE, J. C. R. Fatores críticos no processo de transferência de tecnologia: um estudo de caso do CENPES - Centro de Pesquisa da Petrobras. 2013, 109 p. Dissertação (Mestrado)Departamento de Administração. Pontifícia Universidade Católica do Rio de Janeiro, Rio de Janeiro, 2013.

Perspectivas em Gestão \& Conhecimento, João Pessoa, v. 8, número especial, p. 21-38, out. 2018. 
EIRIZ, V. Proposta de tipologia sobre alianças estratégicas. Rev. Administração Contemporânea, v. 5, n. 2, p. 65-90, 2001. Disponível em: http://www.scielo.br/scielo.php?script=sci arttext\&pid=S141565552001000200004\&lng=en\&nrm=iso. Acesso em: 28 jun. 2018.

EUROPEAN PATENT OFFICE - EPO. Base de dados, Espacenet, 2018. Disponível em: <https://worldwide.espacenet.com/advancedSearch?locale=en_EP>. Acesso em: 28 jun. 2018.

FLORES, C. Segredo industrial e o know-how: aspectos jurídicos internacionais. Rio de Janeiro: Lumen Juris, 264 p., 2008.

HASSANI, H.; SILVA, E. S.; AL KAABI, A. M. The role of innovation and technology in sustaining the petroleum and petrochemical industry. Technological Forecasting \& Social Change, v. 119, 2017, p. 1-17.

HOFFMANN, W. A. M. Monitoramento da informação e inteligência competitiva: realidade organizacional. InCID: R. Ci. Inf. e Doc., Ribeirão Preto, v. 2, n. 2, p. 125-144, jul./dez. 2011.

IFP ÉNERGIES NOUVELLES, 2018. Le groupe IFPEN. Disponível em http://www.ifpenergiesnouvelles.fr/Developpement-industriel/Le-groupe-IFPEN. Acesso em: 28 jun. 2018.

INSTITUTO NACIONAL DA PROPRIEDADE INDUSTRIAL - INPI. Instrução Normativa no 70, de 11 de abril de 2017. Dispõe sobre o procedimento administrativo de averbação de licenças e cessões de direitos de propriedade industrial e de registro de contratos de transferência de tecnologia e de franquia. 2017. Disponível em: http://www.inpi.gov.br/sobre/legislacao1/IN702017.pdf. Acesso em: 28 jun. 2018.

. Base Contratos - Base de dados, 2018. 2018a. Disponível em https://gru.inpi.gov.br/pePl/isp/contratos/ContratoSearchBasico.jsp. Acesso em: 28 jun. 2018.

. Base Patentes - Base de dados, 2018. 2018b. Disponível em https://gru.inpi.gov.br/pePl/jsp/patentes/PatenteSearchBasico.jsp. Acesso em: 28 jun. 2018.

KANTER, R. M. The art of alliances. Harvard Business Review, v. 72, n. 4, 1994, p. 96-108.

LI, L.; QIAN, G.; QIAN, Z. Do partners in international strategic alliances share resources, costs, and risks? Journal of Business Research, v. 66, 2013, 489-498.

PERRONS, R. K. How innovation and R\&D happen in the upstream oil \& gas industry: insights from a global survey. Journal of Petroleum Science and Engineering, v. 124, 2014, p. 301-312.

PETROBRAS. Tecnologia Petrobras 2012. Relatório de tecnologia Petrobras. 2012.

Organograma. 2018a. Disponível em: http://www.petrobras.com.br/pt/quemsomos/organograma/. Acesso em: 25 jun. 2018. 
Estratégia. 2018b. Disponível em: http://www.petrobras.com.br/pt/quemsomos/estrategia/. Acesso em: 28 jun. 2018.

PORTER, M. E. Competitive strategy: techniques of analysing industries and competitors. The Free Press, New York, 397 p., 1980.

SPIANDORELLO, F. M.; HOFFMANN, W. A. M. Contratos internacionais de transferência de tecnologia universidade-empresa: um estudo exploratório. In: ENCONTRO ACADÊMICO DE PROPRIEDADE INTELECTUAL, INOVAÇÃO E DESENVOLVIMENTO - Enapid, 10., Rio de Janeiro: Instituto Nacional da Propriedade Industrial. Anais... 2017, 40 p.

TORDO, S.; TRACY, B. S.; ARFAA, N. National oil companies and value creation. World Bank working paper no 218. The World Bank, 2011, p. 42.

UNITED NATIONS CONFERENCE ON TRADE AND DEVELOPMENT - UNCTAD. Transfer of technology and knowledge sharing for development: science, technology and innovation issues for developing countries. In UNCTAD Current Studies on Science, Technology and Innovation, no 8. Genebra: United Nations Publication, 2014, 63 p. UNCTAD/DTL/STICT/2013/8.

WORLD INTELLECTUAL PROPERTY ORGANIZATION - WIPO. Successful Technology Licensing. $2015,66 \mathrm{p}$.

YUSUF, Y. Y.; GUNASEKARAN, A.; MUSA, A.; DAUDA, M.; EL-BERISHY, N. M.; CANG, S. A relational study of supply chain agility, competitiveness and business performance in the oil and gas industry. Int. J. Production Economics, v. 147, 2014. 531-543.

Artigo recebido em 13/10/2018 e aceito para publicação em 29/10/2018 